\title{
IDENTIFICAÇÃO DE REGIMES FLUIDODINÂMICOS EM LEITO DE JORRO POR MEDIDAS DE FLUTUAÇÃO DE PRESSÃO
}

\author{
M. C. MELO*, C. A. S. FELIPE, C. A. ROSA, I. H. M. MATTOS, R. N. M. RIOS, F. M. BRITO \\ Universidade Federal do Rio Grande, Escola de Química e Alimentos \\ "e-mail: monicacorrea.melo@ hotmail.com
}

\begin{abstract}
RESUMO
A identificação e a distinção de regimes fluidodinâmicos típicos de leito de jorro foram realizadas através da obtenção de sinais de medidas de flutuação de pressão. As séries de tempo obtidas foram tratadas por análise no domínio do tempo, por média e desvio padrão das medidas de pressão, e no domínio da frequência, por Transformada Rápida de Fourier. Buscou-se caracterizar os principais regimes de contato nesse tipo de leito móvel, dando ênfase na frequência dominante e largura dos espectros de potência. Uma coluna cilíndrica de $0,21 \mathrm{~m}$ de diâmetro e de base cônica com inclinação de $60^{\circ}$ foi empregada no estudo, e os sólidos utilizados foram pellets de poliuretano e sementes de soja. As condições experimentais utilizadas nos experimentos corresponderam a uma relação entre a altura do leito fixo e o diâmetro de coluna de 1:1 e 1,5:1; as medidas de flutuação de pressão foram realizadas em um ponto localizado logo acima do orifício de entrada do ar. A obtenção dos dados de flutuação de pressão foi feita utilizando transdutores de pressão ligados ao leito e a um sistema de aquisição de dados; as informações online do leito eram indicadas em uma rotina desenvolvida em software de aquisição de dados. Os parâmetros fluidodinâmicos foram obtidos através das curvas fluidodinâmicas. Por meio da análise espectral foi possível identificar e distinguir os principais regimes fluidodinâmicos em leito de jorro: leito fixo, jorro interno e jorro estável, esse último confirmado por observações visuais.
\end{abstract}

\section{INTRODUÇÃO}

O leito de jorro surgiu em 1954, desenvolvido por Mathur e Gishler para secagem de trigo (MATHUR; EPSTEIN, 1974). Desde a sua concepção, a técnica do leito de jorro vem sendo empregada em uma grande variedade de operações com partículas grossas, isto é, com $d_{p}>1 \mathrm{~mm}$, destacando-se a secagem, recobrimento e granulação de partículas, além de ser um potencial reator químico (XU et al., 2004).
Nos processos realizados em leito de jorro, o melhor rendimento é garantido quando se mantém a estabilidade do regime de contato. Dependendo das aplicações a que se destina, certas exigências devem ser cumpridas para manter o regime operando estavelmente, como, por exemplo, as taxas de transferência de calor e de massa, a homogeneidade do produto desejado, assim como sua integridade física e a relação custo/benefício do equipamento. 
Embora diferentes abordagens e técnicas experimentais venham sendo aplicadas para identificar os regimes de contato gás-sólido em leito de jorro, a análise de medidas de flutuação de pressão tem demonstrado ser uma das mais eficazes (PISKOVA; MÖRL, 2008). Estas medidas resultam na obtenção de séries de tempo de flutuação de pressão cujo conjunto de dados possibilita a identificação de mudanças na fluidodinâmica do leito, como colapso do jorro e também a transição para o jorro instável (indesejável por não apresentar contato homogêneo entre sólidos e gás), e a intervenção nas condições do leito, como temperatura e velocidade do ar, para a manutenção da estabilidade do regime de contato, evitando dessa forma perdas econômicas, em processos de secagem de grãos e pastas e recobrimento de partículas, por exemplo.

Esse estudo teve como objetivo a identificação dos principais regimes fluidodinâmicos de leito de jorro para dois sólidos, sementes de soja e pellets de poliuretano, através de análise no domínio do tempo e da frequência espectral de medidas de flutuações de pressão no leito.

\section{MATERIAIS E METODOLOGIA EXPERIMENTAL}

Os dados experimentais foram obtidos em um leito de jorro cônico-cilíndrico, de $0,21 \mathrm{~m}$ de diâmetro, ângulo da base cônica de $60^{\circ}$ e altura do leito de partículas correspondendo a relação altura de leito fixo/diâmetro da coluna de 1:1 e 1,5:1, ou respectivamente $0,21 \mathrm{~m}$ e $0,315 \mathrm{~m}$.

A unidade experimental era composta pelo leito de jorro, soprador de ar e sistema de aquecimento e resfriamento de ar, transdutores de pressão, termopares, placa de aquisição de dados e computador com software LabVIEW versão 8.5, para tratamento e armazenamento de dados, como mostra a Figura 1.

Figura 1-Unidade experimental.

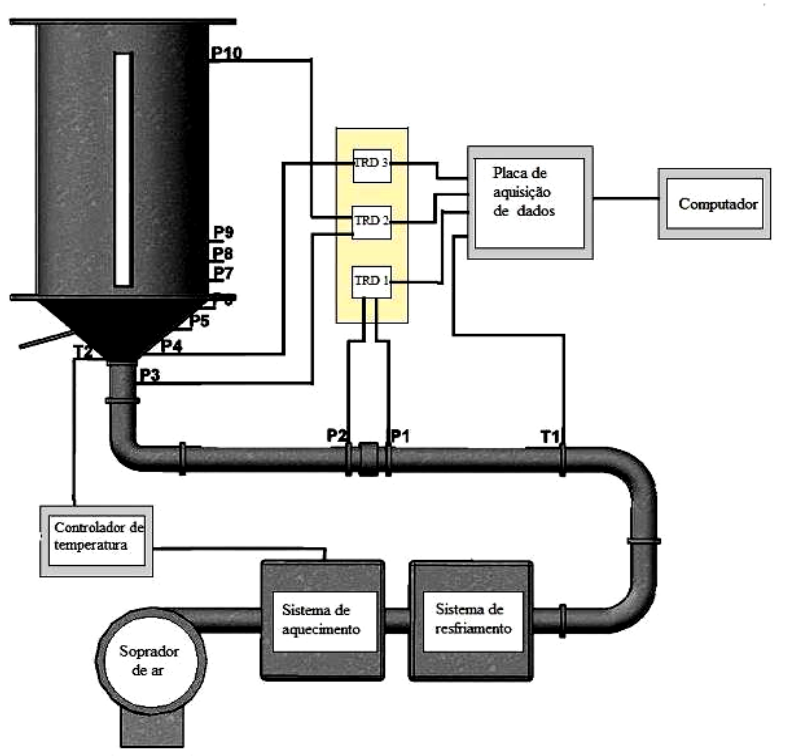

Fonte: Autor (2015).

A caracterização das partículas foi realizada por picnometria, paquimetria, ensaio de proveta e projeção de área das partículas. As partículas utilizadas nesse estudo foram sementes de soja e pellets de poliuretano. Diâmetro de partícula, massa específica, porosidade do leito e esfericidade da partícula são apresentadas na Tabela 1. A picnometria tem por objetivo a determinação da massa específica de líquidos e, de forma indireta, a determinação do diâmetro e massa específica de partículas; a técnica consiste na imersão de uma quantidade conhecida de partículas em um recipiente de volume conhecido (picnômetro), que é posteriormente preenchido por um líquido, usualmente água; o volume de líquido deslocado corresponde ao volume ocupado pelas partículas no picnômetro (CREMASCO, 2012). Para as sementes de soja o volume ocupado por suas partículas no picnômetro foi considerado como o volume de uma amostra de esferas, tendo em vista o formato das sementes de 
soja, obtendo-se de forma indireta o diâmetro da partícula.

A esfericidade, $\varphi$, é o índice que indica o quanto a forma de uma partícula se aproxima à de uma esfera. Sua definição é dada pela razão entre o diâmetro de uma esfera de igual volume ao volume da partícula e o diâmetro da menor esfera circunscrita à área projetada da partícula em repouso (WADELL apud CREMASCO, 2012). Neste trabalho, a esfericidade das partículas foi determinada pelo quociente entre o diâmetro inscrito e o diâmetro circunscrito da projeção da sombra das partículas sobre uma superfície plana e em repouso, Equação 1, conforme técnica citada em Cremasco (2012). Foram projetadas as áreas de 80 partículas para cada sólido, das quais se obtiveram os diâmetros médios inscritos e circunscritos e finalmente, o valor da esfericidade.

$$
\varphi=\frac{\bar{d}_{i n s c}}{\bar{d}_{\text {circ }}}
$$

As curvas fluidodinâmicas foram obtidas nas duas alturas de leito para o registro da velocidade de jorro mínimo e identificação visual do regime de jorro estável.

Tabela 1 - Propriedades das partículas.

\begin{tabular}{ccc}
\hline Partículas & Soja & Poliuretano \\
\hline$d_{p}, \mathrm{~mm}$ & $5,97 * \pm 0,08$ & $3,02 \pm 0,03$ \\
$\rho_{s}, \mathrm{~kg} / \mathrm{m}^{3}$ & 1277,6 & 918,93 \\
$\varepsilon$ & 0,44 & 0,40 \\
$\varphi$ & $0,89 \pm 0,04$ & $0,70 \pm 0,04$
\end{tabular}

* Diâmetros médios obtidos por picnometria em duplicata.

Fonte: Autor (2015).
A análise estatística e a análise espectral das flutuações de pressão nos diferentes regimes foram realizadas em velocidades conhecidas de ar, tendo como referência a velocidade de jorro mínimo. A taxa de aquisição de dados utilizada para a obtenção das curvas fluidodinâmicas e para os espectros de potência foi de $100 \mathrm{~Hz}$, sendo coletados 1024 pontos para a primeira e 8192 pontos para o segundo.

De acordo com XU et al (2004), a análise estatística das medidas de um sinal é a técnica de tratamento dos dados mais simples e mais comumente empregada, pois é de rápida e de fácil aplicação. Os parâmetros estatísticos empregados nesse estudo foram a média e o desvio padrão do sinal de flutuação de pressão, este obtido pela Equação 2.

$$
\sigma=\sqrt{\frac{1}{N-1} \sum_{i=1}^{N}\left(x_{i}-\bar{x}\right)^{2}}
$$

$\mathrm{Na}$ análise no domínio da frequência das medidas de flutuação de pressão em leito de jorro, a distribuição de frequência, expressa na forma de espectros de potência, foi determinada por tratamento matemático logo após a obtenção das séries de tempo, aplicando a Transformada de Fourier, largamente empregada em trabalhos de identificação de regimes de contato em leitos móveis, como em Silva (1998), Xu et al (2004), Lourenço (2006), Oliveira et al (2008), Piskova; Mörl (2008), Butzge (2012) e Mostoufi; Kulah; Koksal (2015). A Transformada de Fourier em um intervalo de tempo finito do registro $\mathrm{x}(\mathrm{t})$ é definida conforme a Equação 3.

$$
X(f, T)=\int_{0}^{t} x(t) e^{-2 j \pi f t} d t
$$

A distribuição de frequência foi expressa em espectros de potência, gerados pelo software LabVIEW logo após cada série 
de tempo amostrada. Como a Transformada Rápida de Fourier (FFT) do sinal real é um número complexo, o espectro retorna uma matriz com as partes positiva e negativa do espectro. Os valores do sinal espectral foram tratados no software pela média RMS (Root Mean Square), seguindo a Equação 4, onde a diferença $t_{2}-t_{1}$ é o tempo de integração.

$x_{R M S}=\sqrt{\frac{1}{\left(t_{2}-t_{1}\right)} \int_{t_{1}}^{t_{2}} x^{2}(t) d t}$

\section{RESULTADOS}

\subsection{Análise no Domínio do Tempo}

No leito de poliuretano, na velocidade de mínimo jorro, observaram-se oscilações na fonte em direção à região anular, ocasionando variação na altura do leito. Atribuem-se essas oscilações ao formato de pellet do sólido e sua massa específica. Para velocidades superiores a 1,1 $v_{j m}$, a fonte passou a ter movimentação homogênea, típica de jorro estável e característica dos sólidos classificados por Geldart no grupo D (considerando sua massa específica e diâmetro de partícula). Algumas séries temporais do poliuretano são demostradas na Figura 2.

Com o aumento na velocidade do ar, as séries de tempo comportaram-se de forma mais aleatória, além de gerarem maiores amplitudes na flutuação de pressão, como visto no comportamento do desvio padrão na Figura 3.

O leito de sementes de soja apresentou fluidodinâmica típica de leito de jorro, devido à esfericidade desse sólido e também a classificação de Geldart dessa partícula no grupo D caracterizado por partículas grandes que apresentam boa fluidodinâmica em leitos de jorro rasos e profundos. Quatro regimes fluidodinâmicos do leito de soja são comparados em séries temporais na Figura 4.
Figura 2- Séries temporais. Regimes: leito fixo $\left(v_{a r}=0,1 v_{j m}\right)$, jorro interno $\left(\mathrm{v}_{\mathrm{ar}}=0,9 \mathrm{v}_{\mathrm{jm}}\right)$, jorro estável $\left(v_{a r}=1,2 v_{j m}\right)$ e jorro instável $\left(v_{a r}=1,5 v_{j m}\right)$. Sólido: poliuretano. $H_{O}=0,21 \mathrm{~m}$.

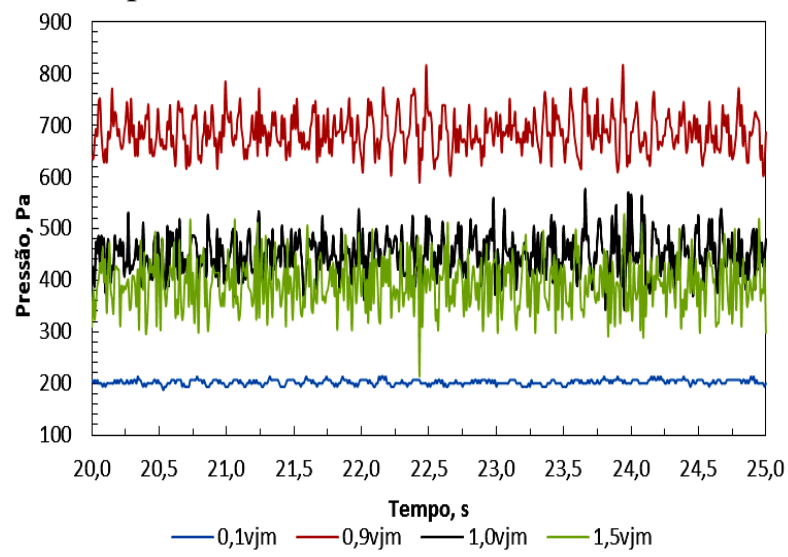

Fonte: Autor (2015)

Figura 3 - Desvio padrão da flutuação de pressão. Sólido: poliuretano.

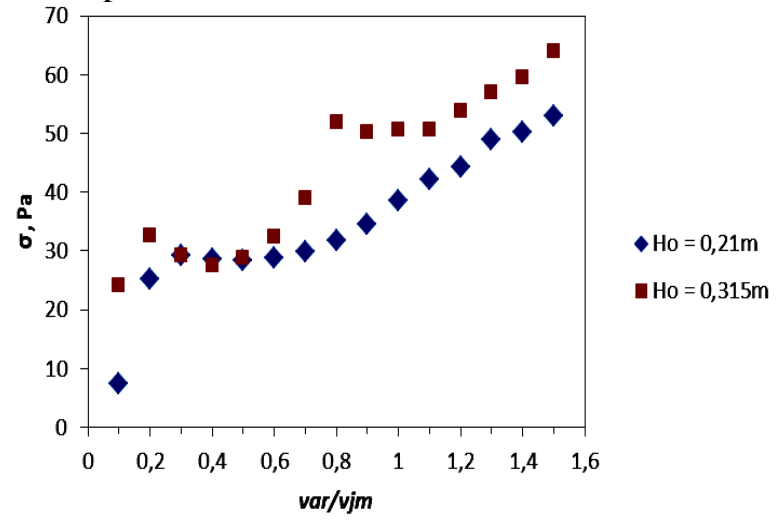

Fonte: Autor (2015)

Figura 4 - Séries temporais. Regimes: leito fixo $\left(v_{a r}=0,1 v_{j m}\right)$, jorro interno $\left(v_{a r}=0,8 v_{j m}\right)$, jorro estável $\left(v_{a r}=1 v_{j m}\right)$ e jorro instável $\left(v_{a r}=1,4 v_{j m}\right)$. Sólido: soja. $H_{O}=0,21 \mathrm{~m}$.

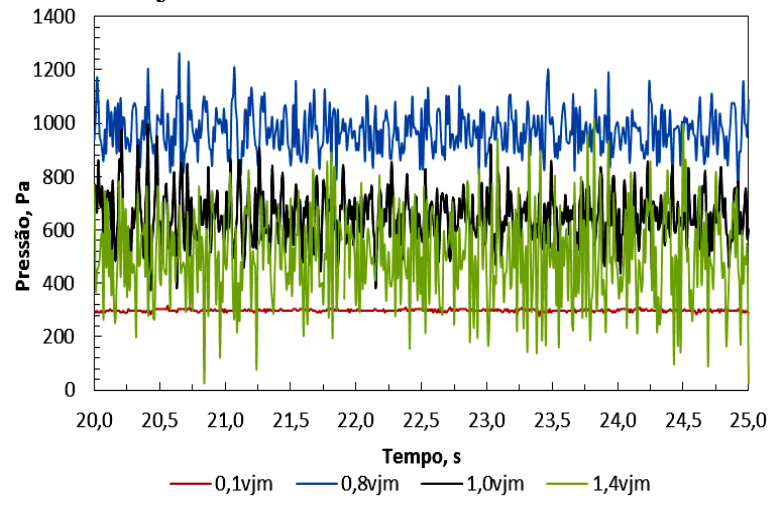

Fonte: Autor (2015) 
O desvio padrão do leito de soja apresentou crescimento mais linear com o aumento da velocidade de ar se comparado ao leito de poliuretano, como observado na Figura 5. Acredita-se que a diferença entre as tendências do desvio padrão dos dois sólidos se deve às características físicas das partículas, tanto massa específica quanto esfericidade, e por consequência às oscilações geradas nos leitos durante a passagem do ar, que foram mais percebidas no leito de poliuretano.

Figura 5 - Desvio padrão da flutuação de pressão. Sólido: soja.

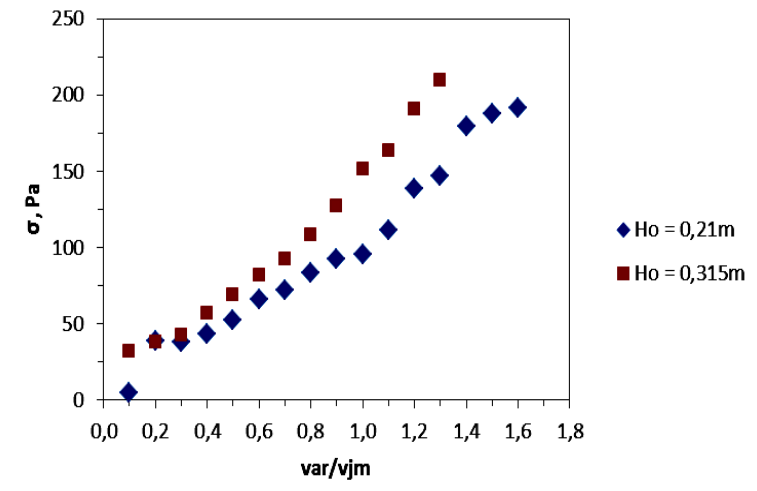

Fonte: Autor (2015)

\subsection{Análise no Domínio da Frequência}

Os espectros de potência das flutuações de pressão no leito do poliuretano revelaram frequência dominante no leito fixo de aproximadamente $7 \mathrm{~Hz}$, que aumentou para 8 $\mathrm{Hz}$ no regime de jorro interno. Para velocidades de ar mais altas, os espectros indicaram distribuição de frequências mais larga, porém a maior componente de frequência no regime de jorro estável manteve-se em $8 \mathrm{~Hz}$, como demonstra a Figura $6(d)$.

$\mathrm{Na}$ altura de $H_{o}=0,315 \mathrm{~m}$, as flutuações de pressão do leito de poliuretano geraram maiores amplitudes de frequência e espectros de potência com uma componente destacada em $7,4 \mathrm{~Hz}$ em leito fixo e de $9 \mathrm{~Hz}$, aproximadamente, em jorro interno e jorro estável, como demonstra a Figura 7.

Figura 6 - Espectros de potência. Sólido: poliuretano. $H_{o}=0,21 \mathrm{~m}$. (a) Leito fixo, (b) Jorro interno, (c) Transição de jorro interno para jorro estável e (d) Jorro estável (mínimo jorro).
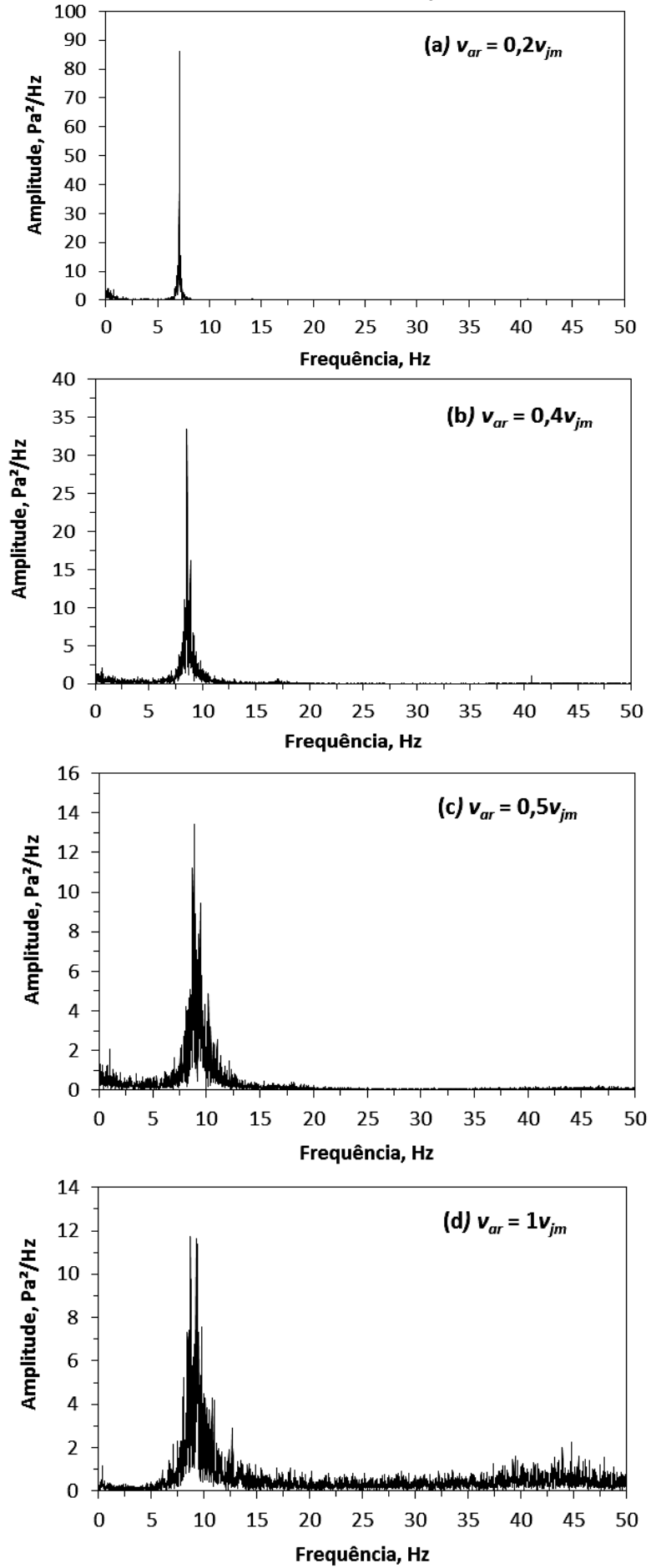

Fonte: Autor (2015). 
Figura 7- Espectros de potência. Sólido: poliuretano. $H_{o}=0,315 \mathrm{~m}$. (a) Leito fixo, (b) Jorro interno, e (c) Jorro estável.
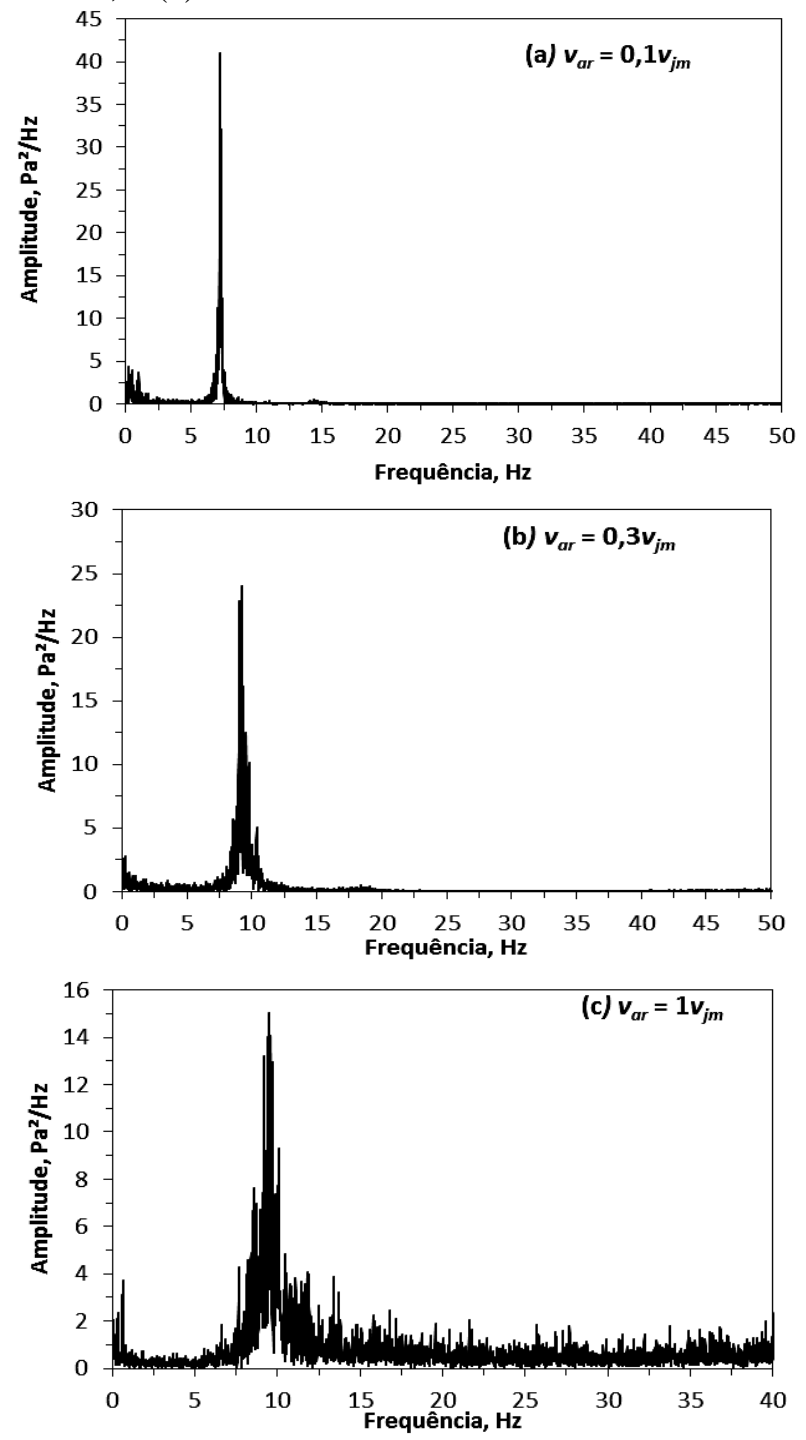

Fonte: Autor (2015).

Os espectros de potência do leito de soja, de uma forma geral, mostraram-se semelhantes aos registrados para o leito de poliuretano, com pico de frequência dominante no leito fixo, alargamento do espectro na transição de jorro interno para estável, além do aumento da frequência dominante, como evidenciado na Figura 8(a) a (c). Já na velocidade de $1,3 v_{j m}$, Figura $8(d)$, pode-se observar a redução da frequência dominante para um valor aproximado de 6 $\mathrm{Hz}$.
Figura 8 - Espectros de potência. Soja. $H_{o}=0,315 \mathrm{~m}$. (a) Leito fixo, (b) Jorro interno, (c) Transição de jorro interno para jorro estável e (d) Jorro estável.
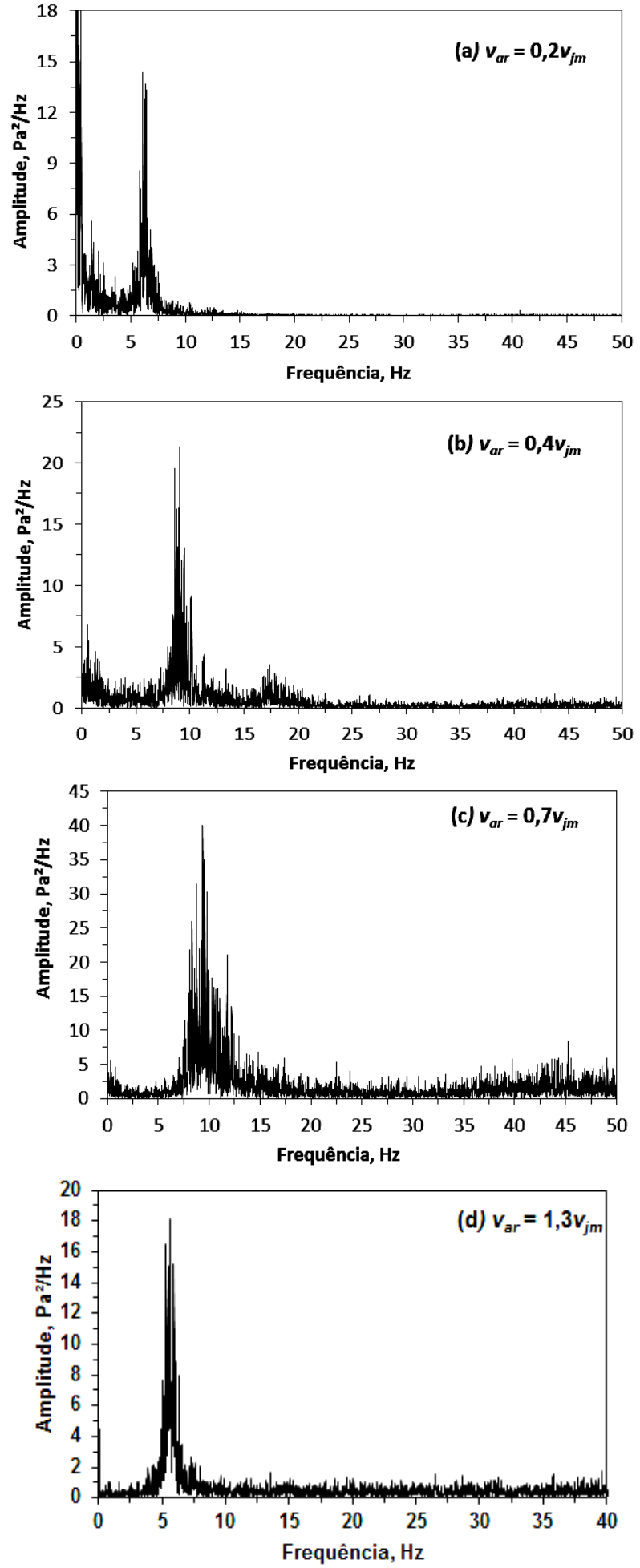

Fonte: Autor (2015). 


\section{CONCLUSÕES}

As sementes de soja e os pellets de poliuretano tiveram comportamento fluidodinâmico típico de leito de jorro, com a formação de jorro estável, que pôde ser identificado visualmente, além de leito fixo e jorro interno. A análise no domínio do tempo possibilitou a verificação do comportamento da amplitude da flutuação de pressão em função da velocidade de ar. $\mathrm{O}$ desvio padrão aumentou gradativamente conforme a velocidade do ar era aumentada, sendo o comportamento esperado para essa configuração de leito móvel. Na maioria dos resultados, obteve-se: frequência dominante no leito fixo, transição de leito fixo para jorro interno com aumento da frequência dominante, transição de jorro interno para jorro estável marcada por espectros mais largos com frequência dominante semelhante à do jorro interno, comportamento que se manteve no jorro estável.

\section{NOMENCLATURA}

$d_{\text {circ }} \quad$ Diâmetro circunscrito da projeção da sombra da partícula, $\mathrm{mm}$

$d_{\text {insc }} \quad$ Diâmetro inscrito da projeção da sombra da partícula, mm

$d_{p} \quad$ Diâmetro de partícula, $\mathrm{mm}$

$f \quad$ Frequência espectral, $\mathrm{s}^{-1}$

$H_{o} \quad$ Altura do leito fixo, $\mathrm{m}$

var Velocidade superficial do ar, $\mathrm{m} / \mathrm{s}$

$v_{j m} \quad$ Velocidade de mínimo jorro, $\mathrm{m} / \mathrm{s}$

$x \quad$ Sinal temporal de flutuação de pressão, $\mathrm{Pa}$ $x R M S \quad$ Média RMS, $\mathrm{Pa}^{2} / \mathrm{Hz}$

$\varepsilon \quad$ Porosidade do leito, adimensional

$\rho_{s} \quad \begin{aligned} & \text { Massa } \\ & \mathrm{kg} / \mathrm{m}^{3}\end{aligned}$

$\sigma \quad$ Desvio padrão, $\mathrm{Pa}$

$\varphi$ Esfericidade da partícula, adimensional

\section{REFERÊNCIAS}

BUTZGE, J. J. Controle do regime fluidodinâmico estável durante o processo de umedecimento de inertes em leito de jorro utilizando análise espectral. Campinas - SP: FEQ/UNICAMP, 2012, 142p.,Dissertação de Mestrado.

CREMASCO, M. A. Operações unitárias
em sistemas particulados e fluidodinâmicos. São Paulo: Blucher, 2012.

LOURENÇO, R. M. Identificação e Monitoramento de Regimes de Escoamento em Leito de Jorro. Uberlândia - MG: FEQ/UFU, 2006,133p., Dissertação de Mestrado.

MATHUR, K. B.; EPSTEIN, N. Spouted beds. New York: Academic Press, 1974.

MOSTOUFI, N.; KULAH, G.;KOKSAL, M. Flow structure characterization in conical spouted beds using pressure fluctuation signals. Powder Technology.v 269, p. 392400, 2015.

OLIVEIRA, W. P.; SOUZA, C.R.F.; LIM, C.J.; GRACE, J.R. Evaluation of flow regimes in a semi-cylindrical spouted bed through statistical, mutual information, spectral and Hurst's analysis. The Canadian 
Journal of Chemical Engineering. v. 86, p. 582-597, 2008.

PISKOVA, E.; MÖRL, L. Characterization of spouted bed regimes using pressure fluctuation signals. Chemical Engineering Science. v 63, p. 2307 - 2316, 2008.

SILVA, V. A. Monitoramento de regimes de contato gás-sólido em leito de jorro cone-cilíndrico por medidas de queda de pressão em tempo real. Campinas - SP: FEQ/ Universidade Estadual de Campinas, 1998, 130p. Dissertação de Mestrado.

XU, J.; BAO, X.; WEI, W.; SHI, G.; SHEN, S.; BI, H. T.; GRACE, J. R.; LIM, C. J. Statistical and frequency analysis of pressure fluctuations in spouted beds. Powder Technology. v. 140, p. $141-154,2004$.

\section{AGRADECIMENTOS}

Agradecemos à Fundação de Amparo à Pesquisa do Estado do Rio Grande do Sul, FAPERGS, pela bolsa de estudos de pósgraduação e à CAPES, pelo suporte financeiro. 